\author{
Military Technical College \\ Kobry El-Kobbah, \\ Cairo, Egypt.
}

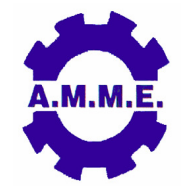

$15^{\text {th }}$ International Conference on Applied Mechanics and Mechanical Engineering.

\title{
INVESTIGATION OF MODERN CONTROL ALGORITHEMS IN MECHATRONIC SYSTEM
}

\author{
H. B. Morsy*, M. M. El-khatib*, W. M. Hussein* and M. H. Mahgoub*
}

\begin{abstract}
The hovercraft is a fascinating mechatronic system that possesses the unique ability to float above land or water. In designing a mechatronic system, there are many factors should be considered, such as mechanical body, control system and actuators, etc.

The objective of the paper is to design, simulate and implement an autonomous model of a small hovercraft. State-space method is used to represent the dynamics of a hovercraft. A real time layered fuzzy navigator for a hovercraft in a dynamic environment is proposed. The system consists of a Takagi-Sugeno-type fuzzy motion planner and a modified proportional navigation based fuzzy controller. MATLAB/Simulink software tool is used to design and verify the proposed algorithm.
\end{abstract}

\section{KEY WORDS}

Mechatronic system, Hovercraft, fuzzy control, Takagi-Sugeno-type fuzzy system.

\footnotetext{
* Egyptian Armed Forces.
} 


\section{NOMENCLATURE}

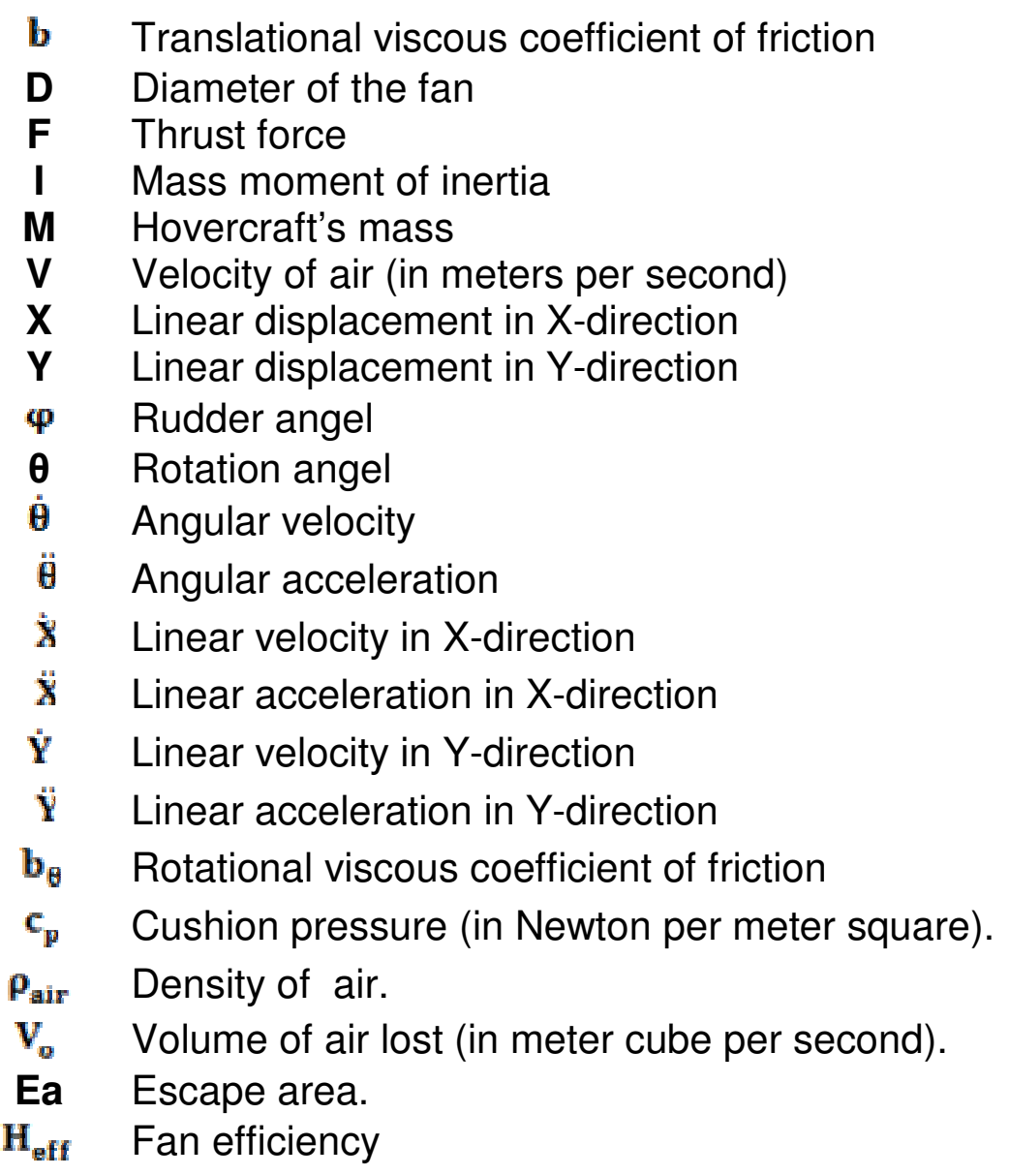

\section{INTRODUCTION}

Hovercraft is an amphibious mean of transportation; it also can be called as AirCushion Vehicles or ACV [1]. The first recorded design project for an air cushion vehicle is in 1716, by a Swedish designer and philosopher, Emmanuel Swedenborg, however the project was not successful and the project was terminated[2,3]. Until mid of $1950 \square \mathrm{s}$, the idea of Christopher Cockerell on first hovercraft that the air will discharge through an angle peripheral jet ring to produce air cushion of hovercraft.

A hovercraft is propelled by an air propeller and supported by a skirt or "cushion" of air retained within a flexible structure. The air inside the cushion is retained by a lift fan, which means that the fan will absorb the air from the environment and compress it into the cushion[4,5]. The hovercraft is used rudder mechanism to steer, therefore the fan must produce the sufficient air flow for the rudder to be effective as a control surface.

The purpose of this paper is to develop and implement an autonomous hovercraft with an intelligent fuzzy control system for steering the proposed system. The system consists of a Takagi-Sugeno-type fuzzy[6] motion planner[7] and a modified proportional navigation based fuzzy controller [8-19]. MATLAB and Simulink, will be 
used in designing the control system and also to verify the proposed system performance.

\section{MAIN PARTS OF HOVERCRAFT MODEL}

From design point of view to make hovercraft model easier and simple, the size of the model must be convenient size to be easy carried and the mass of the model plays an important role in its design, it must be light to use less power motor.

\section{Thrust Mechanism}

A single brushless DC out runner motor is used because it has high power to weight ratio, light weight and less consumption of power due to absence of brushes so there is no maintenance needed. The twin engine craft provides one engine for lift and one for thrust, the needed thrust is obtained by fixation of a propeller on the shaft.

\section{Hovercraft Steering Mechanism}

The hovercraft steering mechanism consists of three rudders to divide the air flow generated from the thrust mechanism to provide the hovercraft with better and easy steering action connected to Servo DC motor while connected to controller [2].

\section{Hovercraft Skirt Design}

The basic idea is that it provides an inflated bubble under the craft It is made from a material that will give way by deforming when it encounters a wave or solid object in its path. Once it has passed over that object the skirt will then reform to its normal shape. In this way it maintains the air below the craft and a good seal with the surface it is passing over. By maintaining the air in the skirt, the energy required to keep the air under the craft is far less the air leakage or loss rate is low when compare to the open peripheral jet as used in SR.N1.This lowering of power requirements due to the change in air loss rate, means that the engine in a skirted hovercraft can be smaller than that used in the peripheral jet (unskirted design)[5]. In fact the increase in load carrying capability of a hovercraft with a skirt is up to ten times higher than one of the same size with no skirt fitted.

\section{Hovercraft Lifting Mechanism}

In a Hovercraft model the air is generated using a fan. This fan generates airflow that creates pressure under the craft as already discussed. The air flow needs vary to meet the criteria of each craft. The basic requirement is to satisfy both the need of air pressure under the craft and an air feed or flow rate that enables the craft to maintain its hover height over varying terrain. As the air is pumped under the craft into the skirt and the plenum chamber it creates, some of it is lost through the gap between the bottom of the skirt and the ground. 


\section{MODEL DESIGN CALCULATIONS}

We have to calculate the air pressure under the hover to lift $[1,2,4]$.This is a function of the craft's weight and its surface area and is found using the following basic calculation[5]:

$$
\text { Air pressure under the hovercraft }=\frac{\text { craft weight }}{\text { surface area }}
$$

The air loss rate is a result of the air gap size and the air velocity due to pressure.

First we will calculate the velocity of the air moving through the gap [5]:

$$
v=\sqrt{\frac{2 c_{p}}{\rho_{\text {air }}}}
$$

where :

$V$ is the velocity of the air $(\mathrm{m} / \mathrm{sec})$

$c_{p}$ is the cushion pressure $\left(\mathrm{N} / \mathrm{m}^{2}\right)$

$\rho_{\text {air }}$ is the air density $\left(\mathrm{Kg} / \mathrm{m}^{3}\right)$

Second step to calculate the escaped area under the hovercraft by the following equation [5]:

$$
\text { Ea }=\text { Air gap } \times \text { Total skirt length }
$$

The volume of the air lost under the hovercraft is given by the following equation [5]:

$$
V_{o}=V \times E_{a}
$$

where:

$$
\begin{aligned}
& V_{o} \text { is the volume of lost air }\left(\mathrm{m}^{3} / \mathrm{sec}\right) \\
& V \text { is the velocity of air }(\mathrm{m} / \mathrm{sec}) \\
& E_{a} \text { is the escaped area }\left(\mathrm{m}^{2}\right)
\end{aligned}
$$

We have to know some important parameters to help us to choose the appropriate fan to use with the model as the running speed of motor, efficiency of fan, the fan characteristics and the power required for the fan. We will show the relations between the parameters to get the required fan as follows [5]:

$$
\text { Power }=\text { Voltage } \times \text { Current }
$$

We have the specification of the fan such as the characteristic of the fan $H_{f}$ which is equal to 8 and the efficiency of fan $H_{e f f}$ which is near to $70 \%$.

Then we can now calculate the diameter of the fan from this formula [6]: 


$$
\text { Diameter }=\left(\frac{{\text { Power } \times \sqrt{H_{f}}}_{\mathrm{rpm}^{3} \times H_{\text {eff }}}}{\mathrm{k}}\right)^{0.2}
$$

where:

$\mathrm{K}$ is the constant $\left(2.1 \times 10^{9}\right), \mathrm{Rpm}$ is the speed of the motor

We have to know some parameters that the pressure provided from the fan has a relation with it as the speed of fan rotation on the tip of the blade. This speed is dependant on the circumference of the fan and the rpm as the form below [5]:

$$
\text { Radial velocity }=\omega \times D=\frac{r p m \times \pi \times D}{60}
$$

where:

$D$ is the diameter

Then the pressure delivered by the fan is [5]:

$$
\text { Pressure }=\left[\frac{\text { Radial velocicy }}{D}\right]^{2}
$$

\section{HOVERCRAFT COMPUTER AIDED DESIGN MODEL}

After the design calculations we need now to design the model of the hovercraft on the computer aided design to help us to know the mass moment of inertia which is accepted for the mathematical model. By using solid edge V20, we review each part and the assembly drawing then we can draw the CAD model of the hovercraft.

\section{Hull}

The hull of the hovercraft consists of a low density balsa construction, the dimensions are $750 \mathrm{~mm} \times 500 \mathrm{~mm} \times 50 \mathrm{~mm}$ and wall thickness is $6 \mathrm{~mm}$. The main forces affected on the hovercraft are the forces and moments exerted by the propulsion unit, the aerodynamic forces and moments exerted by the airflow and the weight force.

\section{Propeller of the Rotor}

The propellers used in the physical model are made ones, thus the model of it is not very accurate, the main forces of propeller are Rotation around the axis of the rotor, this is the main and most obvious motion, the aerodynamic forces and moments exerted by the airflow and finally the weight forces of the propeller which we neglected in our model because it weighs less than 7 grams.

\section{Motor}

Hovercraft model has two DC brushless servo motor one for lift and the other for supporting the thrust force to the hovercraft to move forward. This motor will hold the 
propeller, the DC motor and the propeller will be our propulsion unit and was no need to a hub or a simple gear box since we are using a high torque generating motors compared to its size.

\section{The Mount}

It is a casing which can use all the power of the motor by allowing the air to go inside it in one direction and also can making safe for the propeller.

\section{Assembly Drawing}

Figure 4 shows the assembly of the model and showing the main axes of direction that will be considered while studying the simulink model of the hovercraft. The other components of the hovercraft model such as control circuits, battery, DC motor are acting by the weight force. From the model assembly drawing of the hovercraft and by using the inspection tool of the solid edge V20, we can take the estimated properties of each part such as mass properties as shown in table1 and the mass moment of inertia as shown in table 2.

Table 1. Mass properties.

\begin{tabular}{|c|c|c|c|}
\hline ITEM & Mass(grams) & Quantity & Total Mass \\
\hline Battery & 147 & 2 & 294 \\
\hline Control circuits & 225 & 1 & 225 \\
\hline DC motor & 39 & 2 & 78 \\
\hline ESC & 15 & 2 & 30 \\
\hline Receiver & 40 & 1 & 40 \\
\hline Propeller & 7 & 2 & 14 \\
\hline
\end{tabular}

Table 2. Moment of inertia.

\begin{tabular}{|c|c|}
\hline Axis & Mass Moment of Inertia \\
\hline $\mathrm{X}$ & $0.098562 \mathrm{~kg} \cdot \mathrm{m}^{2}$ \\
\hline $\mathrm{Y}$ & $0.085518 \mathrm{~kg} . \mathrm{m}^{2}$ \\
\hline $\mathrm{Z}$ & $0.016975 \mathrm{~kg} . \mathrm{m}^{2}$ \\
\hline
\end{tabular}




\section{HOVERCRAFT EQUATIONS OF MOTION}

The derivation of equations of motion, the first and most essential step in developing the hovercraft mathematical model, represents the hovercraft movement on a two dimensional plane. The hovercraft has two fans one thrust fan and one lift fan. The thrust fan provided single input F. Figure 5 depicts hovercraft location and orientation $(x, y, \theta)$ along with the sites of possible applied force. The force analysis was possible with derivation from Newton's second law.

$$
\Sigma F=M \times A
$$

where:

$F$ is the applied force

$M$ is the object mass

A the object acceleration

\section{The Analysis in the X-Direction}

The hovercraft frame axis has an angle $\theta$ with the applied force $F$ which can be analyze along the frame axes. Then, using Newton's second law, the acceleration component along the $\mathrm{X}$ - axis can be calculated as followed:

$$
\begin{aligned}
& F \cos \varphi \cos \theta+F \sin \varphi \cos \theta=M \times \ddot{X} \\
& \ddot{X}=\frac{F \cos \varphi \cos \theta+F \sin \varphi \cos \theta}{M}
\end{aligned}
$$

The analysis in the $Y$ direction

$$
\begin{aligned}
& F \cos \varphi \sin \theta-F \sin \varphi \sin \theta=M \times \ddot{Y} \\
& \ddot{\mathbf{Y}}=\frac{F \cos \varphi \sin \theta-F \sin \varphi \sin \theta}{M}
\end{aligned}
$$

\section{The Analysis of Rotational Motion}

Equating the following equation to Newton's $2^{\text {nd }}$ law in terms of rotational motion, where I is the moment of inertia, and is $\alpha$ the angular acceleration as follows:

$$
\sum \mathbf{T}=\mathbf{I} \times \alpha=\mathbf{I} \times \ddot{\theta}
$$

We take the perpendicular distance d (the distance between the applied force and the center of the hovercraft) with the applied force which act as the torque applied on the hovercraft:

$$
\begin{gathered}
I \times \ddot{\theta}=F \sin \varphi \times d \\
\ddot{\theta}=\frac{F \sin \varphi \times d}{I}
\end{gathered}
$$




\section{The Equation of Motion with Friction}

Because the hovercraft rides on a cushion of air and has little contact with the ground, only viscous friction (fluid friction or in this case ground resistance) was considered. Including the hovercraft had a viscous friction in the opposite direction to the hovercraft's motion represented by $F_{\mathrm{f}}$. The friction force is defined by:

$$
\mathbf{F}_{\mathbf{f}}=\mathbf{b} \times \mathbf{N}=\mathbf{b} \times \mathbf{m} \times \mathbf{g}
$$

where :

$\mathrm{b}$ is the coefficient of friction.

$\mathrm{N}$ is the difference between hovercraft's weight and lift force.

$m$ is the hovercraft's mass.

$\mathrm{g}$ is specific gravity.

\section{The Analysis in the X- Direction}

The viscous force is proportional to the hovercraft's velocity, giving the relationship $F_{f}=-b u$,

$$
\begin{aligned}
& F \cos \varphi \cos \theta+F \sin \varphi \cos \theta-b u=m \times \dot{u} \\
& \dot{\mathbf{u}}=\frac{F \cos \varphi \cos \theta+F \sin \varphi \cos \theta-b u}{m}
\end{aligned}
$$

The Analysis in the Y- Direction

$$
\begin{gathered}
F \cos \varphi \sin \theta-F \sin \varphi \sin \theta-b v=m \times \dot{v} \\
\dot{v}=\frac{F \cos \varphi \sin \theta-F \sin \varphi \sin \theta-b v}{m}
\end{gathered}
$$

\section{The Analysis of Rotational Motion}

Equating the following equation to Newton's $2^{\text {nd }}$ law in terms of rotational motion where $\mathrm{I}$ is the moment of inertia, and $\dot{\omega}$ is the angular acceleration, the rotational coefficient of viscous friction $b_{\theta}$ is said to relate viscous torque to angular velocity, then we calculate the angular acceleration from the difference between the component of the applied torque and rotational viscous friction as follows:

$$
\begin{aligned}
& \mathrm{dF} \sin \varphi=\mathrm{I} \dot{\omega} \\
& \dot{\omega}=\frac{\mathrm{dF} \sin \varphi-b_{\theta} \omega}{\mathrm{I}}
\end{aligned}
$$

\section{Defining Parameters for the Modeling}

The viscous translational coefficient of friction was estimated with an experiment governed by the differential equation:

$$
m \ddot{x}=-b \dot{x}
$$


$\dot{\mathrm{x}}=\mathrm{v}$

$\dot{v}+\frac{b v}{m}=0$, let $v=e^{\lambda t}$ then $v=e^{\lambda t}$, substitute in the previous equation we get:

$\lambda e^{\lambda t}+\frac{b}{m} e^{\lambda t}=0$, then $\lambda=-\frac{b}{m}$, then the solution is $v=v_{a} e^{-\frac{b t}{m}}$

while rotational motion was governed by:

$$
\text { I } \ddot{\theta}=-b_{\theta} \theta
$$

$\dot{\theta}=\omega$

$\dot{\omega}+\frac{b_{g} \dot{\omega}}{t}=0$, let $\omega=e^{\lambda t}$ then $\dot{\omega}=e^{\lambda t}$, substitute in the previous equation we get: $\lambda e^{\lambda t}+\frac{b_{\theta}}{I} e^{\lambda t}=0$ then $\lambda=-\frac{b_{\theta}}{I}$.

With a final velocity (v) equal to zero, velocity descends exponentially from max towards zero over time. By substituting $5 \tau$ for time to stop ( $\tau$ in this case equal $\mathrm{m} \backslash$ $b)$, it can be solved for the translational viscous coefficient $b \approx \frac{5 \mathrm{~m}}{\mathrm{~T}_{\mathrm{atap}}}$.

By bringing the hovercraft to a determined top speed in a straight line and allowing it to stop, $\mathrm{T}_{\text {stop }}$ can be measured and $\mathrm{b}$ can be estimated.

The subsequent Fig.6, plots $\mathrm{v}=\mathrm{v}_{\mathrm{s}} \mathrm{e}^{-\frac{\mathrm{bt}}{\mathrm{m}}}$ for the hovercraft. Choosing $5 \mathrm{~T}$ to represent stopping time proved to be reasonable, the stopping time experimentally was 15 seconds, a point at which the plot shows a velocity close to zero, the mass of hovercraft which calculated from the sum of each component's weight was $0.681 \mathrm{~kg}$. By substituting, the measured translational viscous coefficient was $0.227 \mathrm{~kg} / \mathrm{sec}$.

Determining the rotational coefficient of viscous friction was accomplished with a very similar method except that instead of the hovercraft beginning with a maximum translational velocity, it begins the experiment with its greatest rotational velocity according to the Fig .7, $\mathrm{b}_{0} \approx \frac{5 \mathrm{I}}{\mathrm{T}_{\mathrm{stap}}}$.

The moment of inertia was estimated by using the inspection tool of the solid edge in the rotational axes $\mathrm{x}, \mathrm{y}, \mathrm{z}$ were respectively $\left(0.098562 \mathrm{~kg} \cdot \mathrm{m}^{2}, 0.085518 \mathrm{~kg} \cdot \mathrm{m}^{2}\right.$, $0.016975 \mathrm{~kg} \mathrm{~m}^{2}$ ), and we take the value of the moment of inertia in the $z$ axis which is the rotational one, the stopping time experimentally was 20 second. A point at which the plot shows a angular velocity close to zero, then the measured rotational viscous coefficient was $0.004 \mathrm{~kg} \cdot \mathrm{kg} \cdot \mathrm{m}^{2} / \mathrm{sec}$.

\section{STATE SPACE MODELING}

State-space models represent the dynamics of physical systems described by a series of first order coupled differential equations. In the general state-space model, $\mathrm{x} \square R^{n}$ is the state vector and $\mathrm{y}$ is the output. The set of equations is given by: 


$$
\begin{aligned}
& x=A x+B u \\
& y=C x+D u
\end{aligned}
$$

The column vector ' $x$ ' is called the state of the system. The state of the system is a set of variables describe the future response of a system, given the present state, the excitation inputs and the equations describing the dynamics. For mechanical systems, the state vector elements usually consist of the positions and velocities of the separate bodies, as a result let:

$$
\begin{aligned}
& \dot{x}=\boldsymbol{u} \\
& \dot{y}=v \\
& \dot{\theta}=\omega \\
& \dot{\mathbf{u}}=\frac{F \cos \varphi \cos \theta+F \sin \varphi \cos \theta-b \mathbf{u}}{\mathbf{m}} \\
& \dot{\mathbf{v}}=\frac{F \cos \varphi \sin \theta-F \sin \varphi \sin \theta-\mathbf{b v}}{\mathbf{m}} \\
& \dot{\omega}=\frac{\mathrm{dF} \sin \varphi-b_{\theta} \omega}{\mathbf{I}}
\end{aligned}
$$

we put the values of the rows and columns of the matrix $A$ from the previous equations described the velocity and we take the second part of the equations which described the effect of the viscous friction on the system and we make some assumptions for the linearization for the system that made the value of the angle of the system is so small and near to be equal zero $(\theta \approx 0)$ as in Fig. 8 , the value of $(\cos \theta=1)$ and the value of $(\sin \theta=0)$, then the equations are:

$$
\begin{gathered}
\dot{\mathbf{u}}=\frac{\mathrm{F}-\mathrm{bu}}{\mathrm{m}} \\
\dot{\mathbf{v}}=\frac{-\mathbf{b v}}{\mathrm{m}} \\
\dot{\omega}=\frac{\mathrm{dF}-\mathbf{b}_{g} \omega}{\mathrm{I}}
\end{gathered}
$$

we can write the equations in the state space representation form as follows: 


$$
\left[\begin{array}{c}
\dot{x} \\
\dot{y} \\
\dot{\theta} \\
\dot{u} \\
\dot{v} \\
\dot{\omega}
\end{array}\right]=\left[\begin{array}{cccccc}
0 & 0 & 0 & 1 & 0 & 0 \\
0 & 0 & 0 & 0 & 1 & 0 \\
0 & 0 & 0 & 0 & 0 & 1 \\
0 & 0 & 0 & -b / m & 0 & 0 \\
0 & 0 & 0 & 0 & -b / m & 0 \\
0 & 0 & 0 & 0 & 0 & -b_{\theta} / I
\end{array}\right]\left[\begin{array}{l}
x \\
y \\
\theta \\
u \\
v \\
\omega
\end{array}\right]+\left[\begin{array}{c}
0 \\
0 \\
0 \\
\frac{1}{m} \\
0 \\
\frac{\mathrm{d}}{I}
\end{array}\right][F]
$$

In the previous state space representation we show the relations between the position of the hovercraft and its velocity with the effect of the input force which act on the rudder to guide the hovercraft in the active motion and its turning to right and left and we have to show also the nyquist plot, While nyquist is a graphical technique, it only provides a limited amount of intuition for why a system is stable or unstable, or how to modify an unstable system to be stable as shown in Fig .9.

\section{HOVERCRAFT SIMULINK MODEL}

The simulation of the power system is the important means to study the dynamic performance of the power system of hovercrafts. The simulation model as shown in fig. 10 , is established based on matlab/simulink with the method of system simulation. Through selecting of typical operating conditions and setting of parameters the simulation model and program are checked and debugged and then the simulation calculations of dynamic performance under typical conditions are made.

\section{FUZZY LOGIC IN AUTONOMOUS NAVIGATION}

A number of different strategies has been published in the use of fuzzy logic in autonomous navigation[8]. The use of fuzzy logic in mobile robotics was reported in 1985 by Sugeno and Nishida [9] who developed a fuzzy controller capable of driving a model car along a track delimited by two walls. In 1988, Tekeucho et al [10] published a simple fuzzy logic based obstacle avoidance algorithm. The behavioural based structure has been used in [11-13], in which different behaviours are assigned for different situations. However, the increased level of behaviours employed is accompanied by an increase in complexity of the resulting system. As the number of system variables increases, the number of rules in the conventional complete rule set increases exponentially. The concept of "hierarchical structure" is used $[14,15]$ to tackle the problem wherein the number of rules increase linearly (not exponentially) with the number of the system variable. Unfortunately, the structure needs more training and additional algorithms for tuning the fuzzy parameters. All the behaviours discussed above can be considered as simple, in that they only take into account one single objective. However, to perform autonomous navigation, the robot should be able to perform complex tasks that take multiple objectives into account; for example, going to a goal while avoiding unforeseen obstacles in real time.

Fuzzy controllers are typically designed to consider one single goal [16]. If we want to consider several interacting goals, we have two options. We can write different sets of simpler rules, one specific to each goal, and combine their outputs in some way. This will increase the hardware requirements of the system. Alternatively we 
can write one set of complex rules whose antecedents consider both goals simultaneously. Defining rules for such a system leads to more efficient hardware implementation. This paper focuses on the design of a fuzzy navigator for real time motion in a dynamic environment based on a complex rule base inspired by human capabilities.

\section{Design and Simulation of the Layered Fuzzy Navigator}

Intelligent robotic systems should take task-level commands directly without any planning-type decomposition [17,18]. Additionally it is desirable to design them for a large class of tasks rather than a specific task. As a result, the spilt between vehicle controller design and vehicle action planning is critical since they usually have two different reference bases. The action planning is carried out in terms of events, whereas the execution of planned actions uses a reference frame on existing vehicle control system using a time based or clocked trajectory. The presented system consists of two subsystems, a fuzzy motion planner (FMP) and a modified proportional navigation (PN) based fuzzy controller as illustrated in Fig. 11.

The data from the three sensors plus the direction to the goal are input to the motion planner resulting is a clear direction to move in. Next, the fuzzy controller decides the direction to steer the hovercraft to the goal using the proportional navigation trajectory avoiding the obstacles. The signal processing unit is responsible for calculating the line of sight rate of change and the required steering angle to the goal.

The system philosophy is inspired by human routing when moving between obstacles based on visual information including right and left views to identify the next step to the goal in obstacle-free space. This is analogous to the proposed robot moving safely in an environment based on data "visible" with three ultrasound range finders. These sensors are mounted to the front, right and left of the hovercraft as shown in Fig. 12.

The sensors are modeled such that they scan an array of zeros representing the clear environment with obstacles set to ones if present. They scan the array in a $50^{\circ}$ sector along each sensor axis direction such that it returns the range of the nearest obstacle as shown in Fig.12. The maximum range is set to 5 meters. These specifications meet commercial sensors specifications such as Ultrasonic range finder SRF04, SRF05 and SRF10 [18].

A Sugeno-type [6], fuzzy motion planner of four inputs one output is introduced to give an obstacle-free direction to the robot controller. The inputs are the frontal, right and left obstacle ranges found by the front, right and left sensors. The last input is the angle to the goal, which indicates the difference in direction between the goal and the robot's current direction. The output is an error angle representing the angle needed to go from the robot's direction to a free (suggested) direction. Fuzzy membership functions are assigned to each input as shown in Fig.13.

The membership functions describe the ranges from the sensors designed to give the planner early warning. The left and right sensors assign the obstacle as $\mathrm{N}$ (near) or VN (very near) for the front sensor if its range approaches $90 \%$ of the sensor 
maximum range. For example: the obstacle is near if its range is about 4.5 meter as the sensor maximum range is 5 meter. This enables the planner to determine the feasible trajectory at an early stage to avoid cul-de-sacs.

The fuzzy motion planner uses Takagi-Sugeno fuzzy inference for the rule evaluation $[6,17,18]$. Unlike other fuzzy control methods [19], the Takagi-Sugeno results in the output of a control function for the system depending on the values of the inputs which is ideal for acting as an interpolation supervisor of multiple linear controllers that are to be applied, respectively, to different operating conditions of a dynamic nonlinear system as in the present case. The output of the planner is the angle to an obstacle-free direction[20]. This goes to the second stage to control the robot direction, described by five membership function as shown in table 3 . In order to optimize the membership function width the Whole Overlap, WO, index is calculated from the following equation [16]:

$$
W O=\frac{\int_{x} \min \left(\mu_{1}(x), \mu_{2}(x)\right)}{\int_{x} \max \left(\mu_{1}(x), \mu_{2}(x)\right)}
$$

where:

$\mu_{1}(x)$ and $\mu_{2}(x)$ are two adjacent membership functions.

A low WO (about 14\%) improves the steady state error and response time [16]. Similarly, the LOS changing rate is fuzzified by seven membership functions.

The rules were formulated one-by-one according to the flow chart Fig.14, and then the whole rules-set was analyzed to make it :

- Complete: any combination of the inputs fired at least one rule.

- Consistent: contains no contradictions.

- Continuous: has no neighboring rules with output fuzzy sets that have an empty intersection.

Table 3. The fuzzy membership function for the output of the Sugeno-type fuzzy motion planner.

\begin{tabular}{|c|c|c|c|}
\hline $\begin{array}{c}\text { MF } \\
\text { Name }\end{array}$ & Type & Value & Description \\
\hline SL & Const. & 0.523 & Small to the left (30o) \\
BL & Const. & 0.785 & Big to the left (450) \\
SR & Const. & -0.523 & Small to the right(30o) \\
BR & Const. & -0.785 & Big to the right (45o) \\
F & Const. & 0 & Forward (no change) \\
GOAL & Linear & $4^{\text {th }}$ input & The same angle to the goal as the 4th input \\
\hline
\end{tabular}


Notice that the rules are processed in parallel. The system was modeled and simulated using Simulink, for a given data set of random environments. Different environments with different obstacle distributions are used to test the system performance as shown in Fig.15. The simulation reveals that the system gives good results for non-complicated environments; for example when there is a limited number of obstacles. This is predictable because of the robot's kinematics constraints and constant speed's manoeuvre limitation.

\section{CONCLUSION}

This paper represented a simple approach for the design and implementation of an autonomous hovercraft. There are many factors have been considered, mechanical structure, skirt design, control as well as structure, thrust, drag, etc. The estimated mass and inertial properties from the CAD model were very close to the real model. State-space method has been used to signify the dynamics of the system. A real time layered fuzzy navigator is proposed for the system in a dynamic environment. The system consists of two layers: a Takagi-Sugeno-type fuzzy motion planner; and a modified proportional navigation based fuzzy controller. Simulation results show that the ability of the system to cope with obstacle avoidance as well goalapproaching using a proportional navigation path accounting for hovercraft kinematics.

\section{REFERENCES}

[1] Cheng-long, W., et al., "Amphibious Hovercraft Course Control Based on Adaptive Multiple Model Approach," Proceedings of the 2010 IEEE International Conference on Mechatronics and Automation, Xi'an, China. p. 601-604 (2010).

[2] Yun, L. and A. Bliault, "Theory and Design of Air Cushion Craft", New York, NY, USA: John Wiley \& Sons Inc.(2000).

[3] Aguiar, A.P., L. Cremean, and J.P. Hespanha, "Position Tracking for io Nonlinear Underactuated Hovercraft: Controller Design and Experimental Results," Proceedings of the 42nd IEEE Conference on Decision and Control, Maui, Hawaii USA. p. 3858 - 3863 (2003).

[4] Zhao, J. and J. Pang, "Trajectory Control of Under actuated Hovercraft," Proceedings of the 8th World Congress on Intelligent Control and Automation, Jinan, China. p. 3904-3907 (2010).

[5] Kelleher, E.A., "A STUDY OF A SKIRTLESS HOVERCRAFT DESIGN," DEPARTMENT OF THE AIR FORCE, AIR FORCE INSTITUTE OF TECHNOLOGY Ensign, Ohio p.(2004).

[6] Walker, K. and A.C. Esterline. "Fuzzy motion planning using the TakagiSugeno method," Proceeding of the IEEE Southeastcon, Nashville, TN, USA (2000).

[7] Gupta, K. and A.P. Pobil, "Practical Motion Planning in Robotics: Current approach and Future Directions," John Wiley \& Sons Ltd (1998).

[8] Precup, R.-E. and H. Hellendoorn, "A survey on industrial applications of fuzzy control," Computers in Industry, 62: p. 213-226 (2011). 
[9] Sugeno, M. and M. Nishida, "Fuzzy Control of Model Car," Fuzzy Sets and Systems, 16(2): p. 103-113 (1985).

[10] Takeuchi, T., Y. Nagai, and N. Enomoto, "Fuzzy Control of a Mobile Robot for Obstacle Avoidance," Information Sciences, 45(2): p. 231-248 (1988).

[11] Parasuraman, S., V. Ganapathy, and B. Shirinzadeh, "behaviour based mobile robot navigation by Al techniques: Behavior selection and resolving behavior conflicts using a-level fuzzy inference system," ICGST International Conference on Automation, Robotics and Autonomous Systems (ARAS-05) Cairo, Egypt (2005).

[12] Vadakkepat, P., et al.,"Fuzzy Behavior-Based Control of Mobile Robots," IEEE Transactions on Fuzzy Systems, 12(4): p. 559-564 (2004).

[13] Seraji, H. and A. Howard, "Behavior-Based Robot Navigation on Challenging Terrain: A Fuzzy Logic Approach," IEEE Trans. on Robotics and automation, 18(3): p. 308-321 (2002).

[14] Lin., W.S., C.L. Huang, and C.M. K., "Hierarchical Fuzzy Control for Autonomous Navigation of Wheeled Robots," IEE Proceedings - Control Theory and Applications, 125(5): p. 598-606 (2005).

[15] Hagras, H.A.," A Hierarchical Type-2 Fuzzy Logic Control Architecture for Autonomous Mobile Robots," IEEE Transactions on Fuzzy Systems, 12(4): p. 524-539 (2004).

[16] Reznik, L., "Fuzzy Contollers", Newnes (1997).

[17] Olajubu, E.A., O.A. Ajayi, and G.A. Aderounmu, "A fuzzy logic based multiagents controller," Expert Systems with Applications, p. 4860-4865 (2011).

[18] http://www.robot-electronics.co.uk, Advanced Electronics for Robotics.

[19] Eslami, J.J.B.a.E., "An Introduction to Fuzzy Logic and Fuzzy Sets," PhydicaVerlag Heidelberg (2002)

[20] Call, B.R., "OBSTACLE AVOIDANCE FOR UNMANNED AIR VEHICLES," Department of Electrical and Computer Engineering, Brigham Young University (2006). 


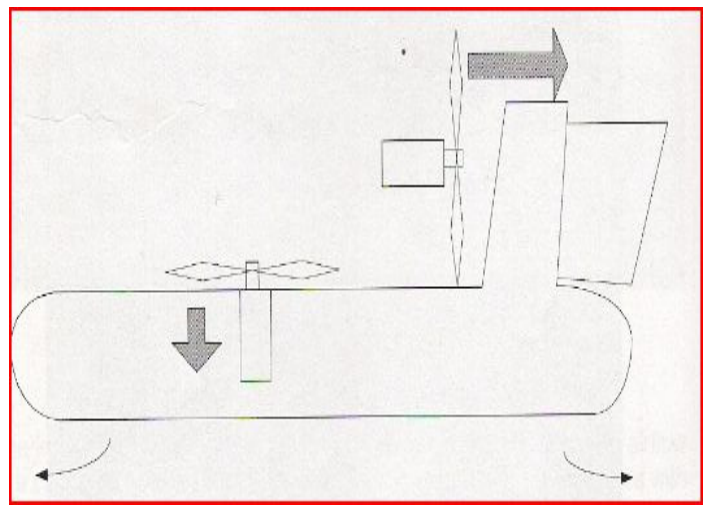

Fig. 1.Twin engine.

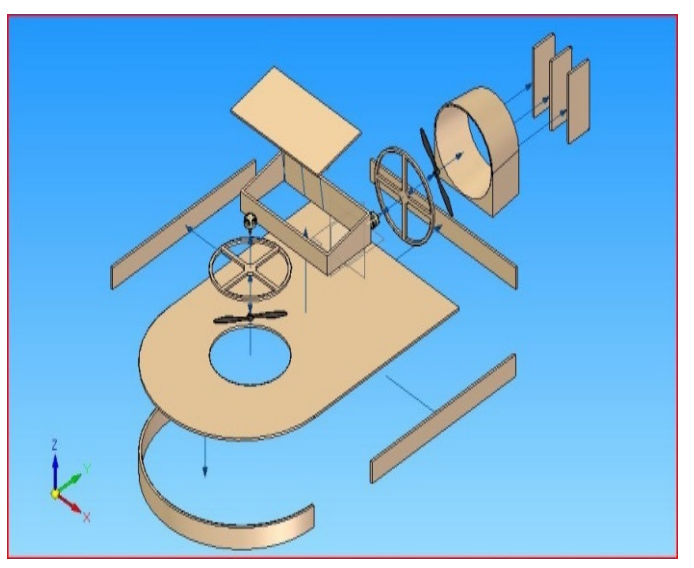

Fig. 3. hovercraft parts.

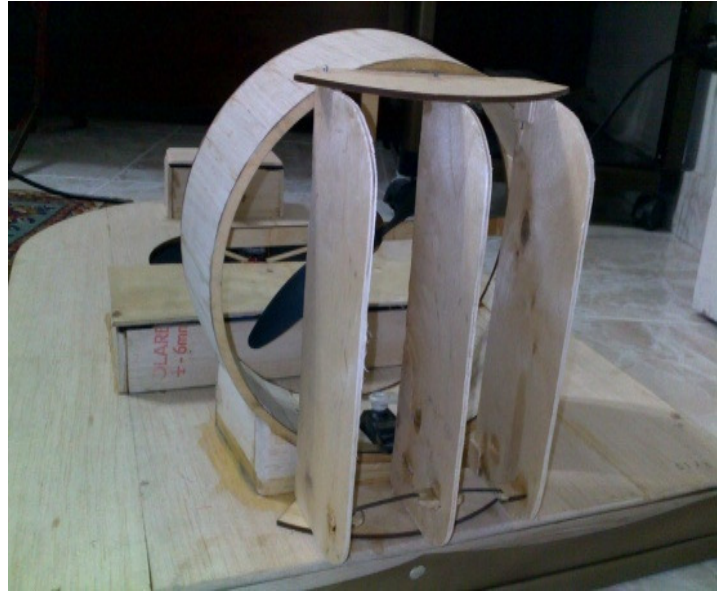

Fig. 2. Hovercraft steering rudders.

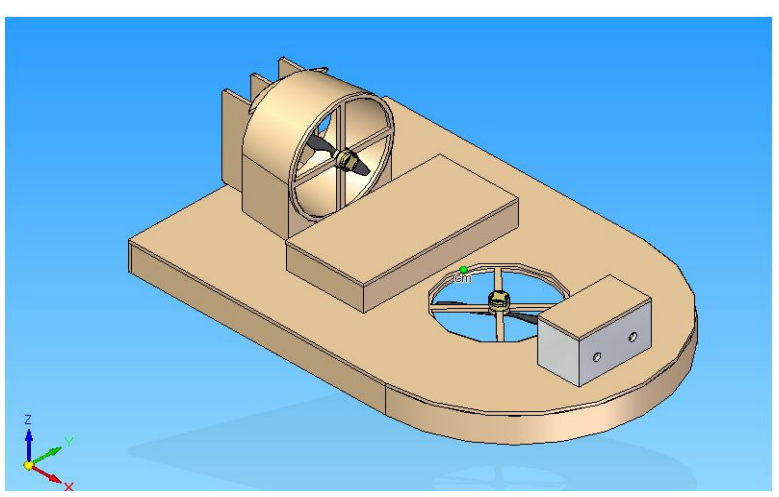

Fig. 4. hovercraft assembly.

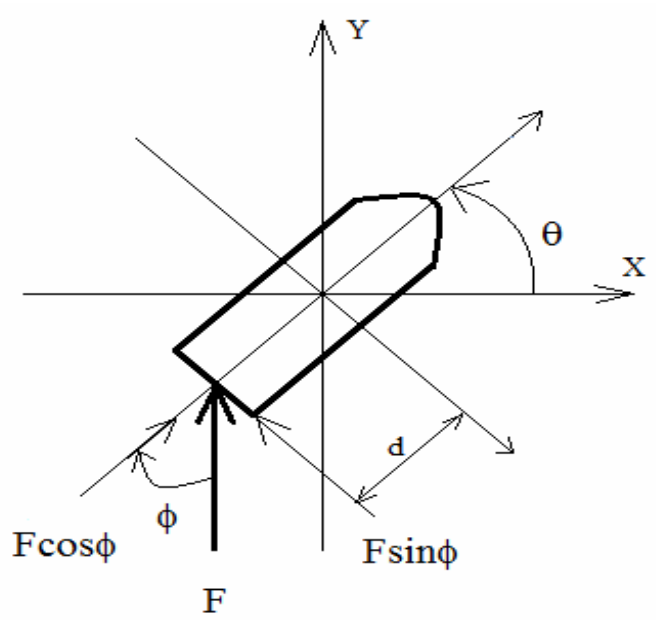

Fig. 5. Forces Analysis on Hovercraft. 


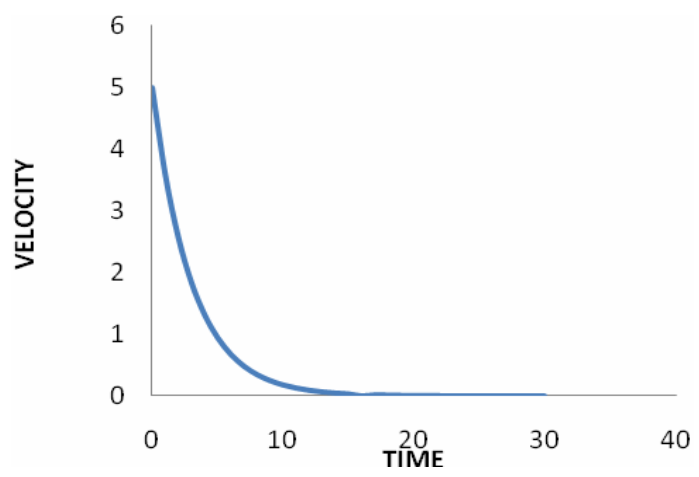

Fig. 6. The velocity $\square$ Time curve.

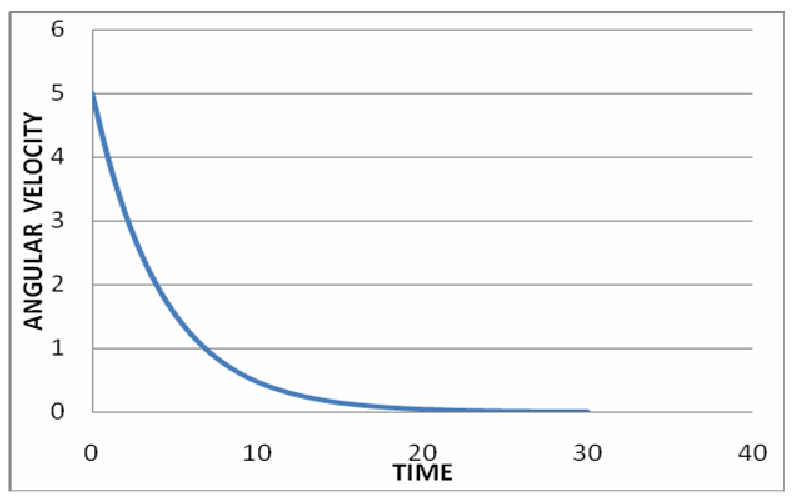

Fig. 7.The Angular Velocity $\square$ Time.

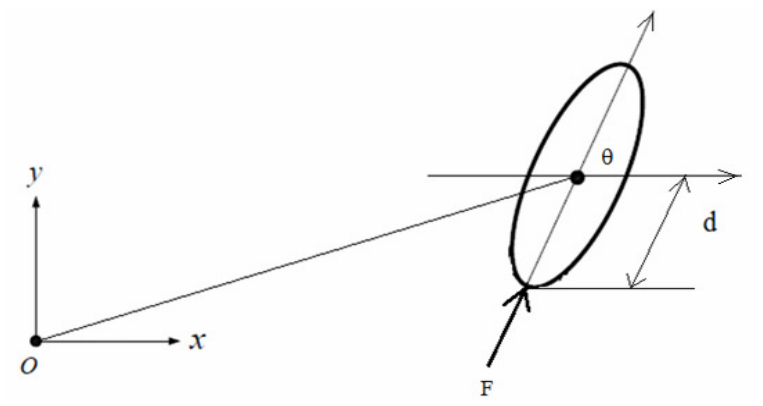

Fig. 8. Forces analysis(friction case)

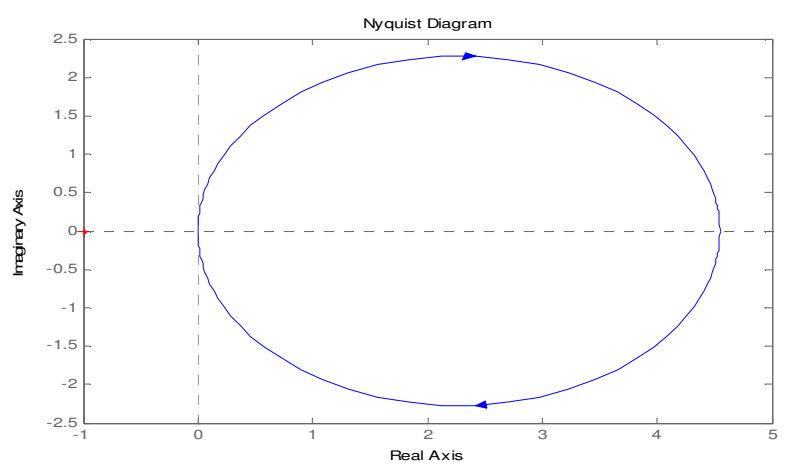

Fig. 9. Nyquist plot. 


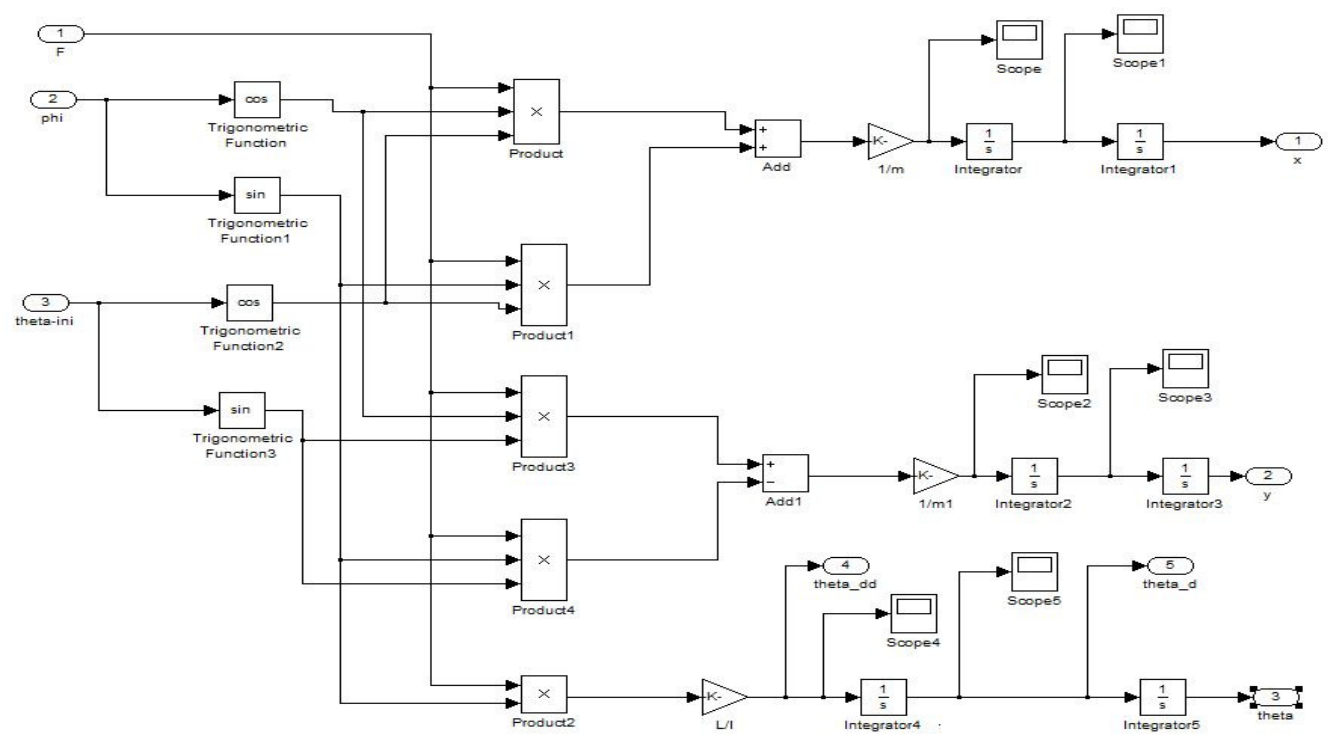

Fig.10. Hovercraft simulink subsystem.

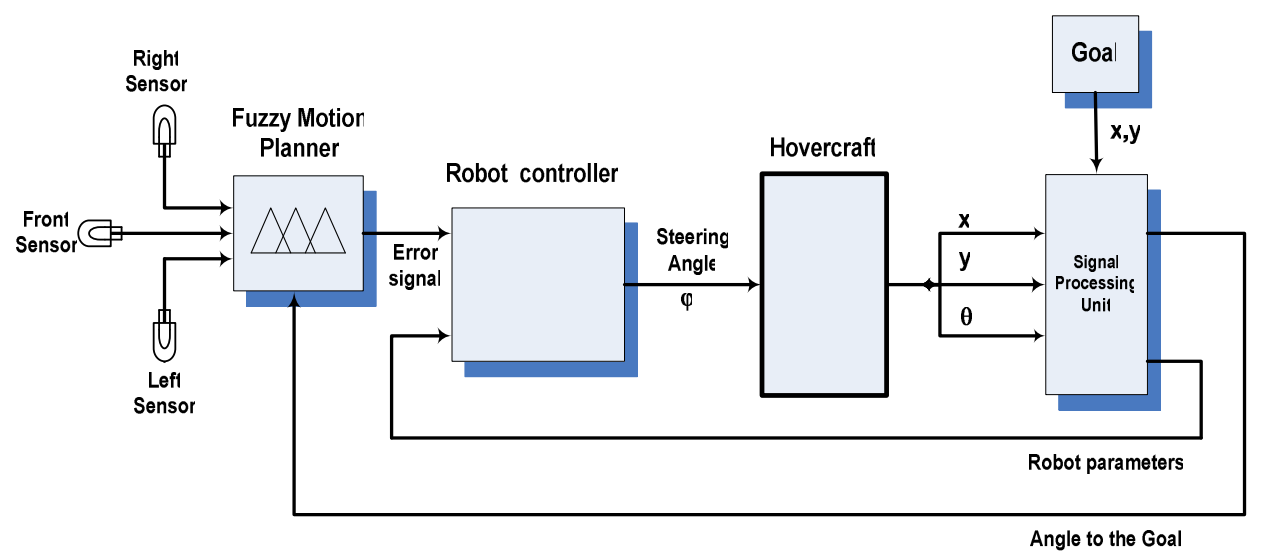

Fig.11. The complete system of fuzzy motion planner.

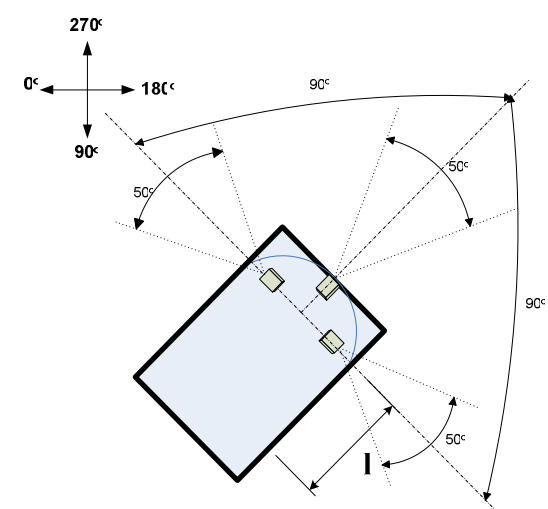

Fig.12. The Robot Sensors with their beam pattern. 

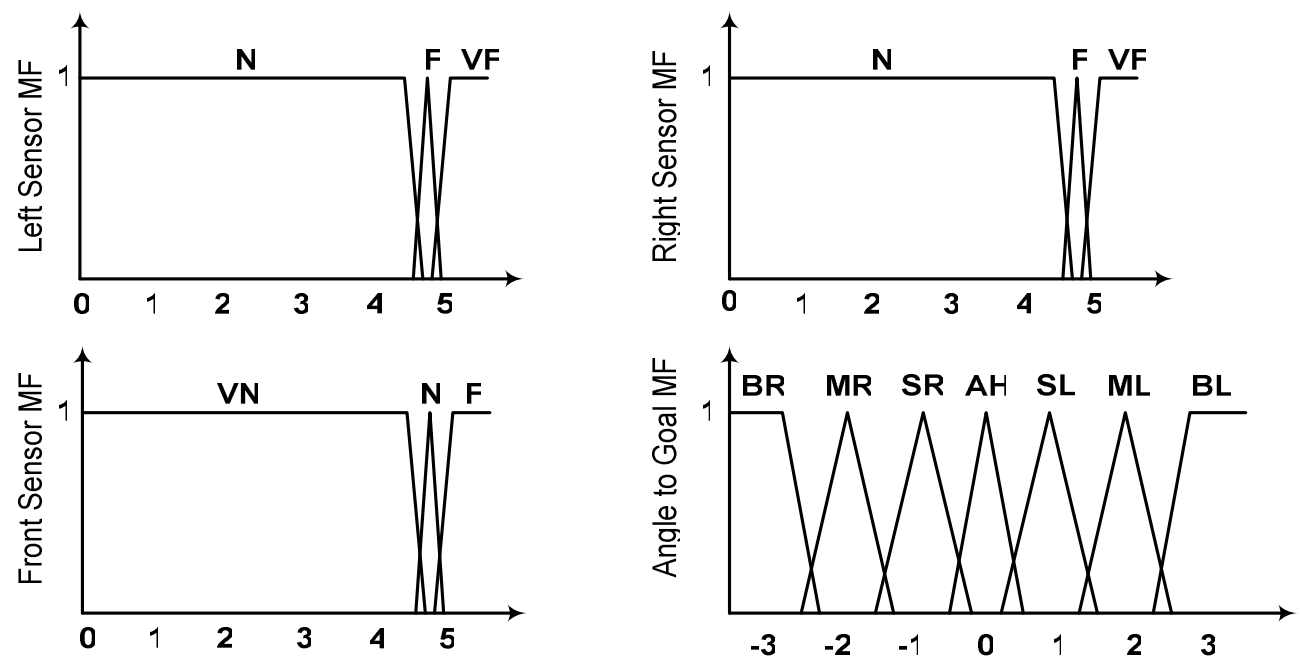

Fig.13. The Membership functions of the fuzzy motion planner inputs.

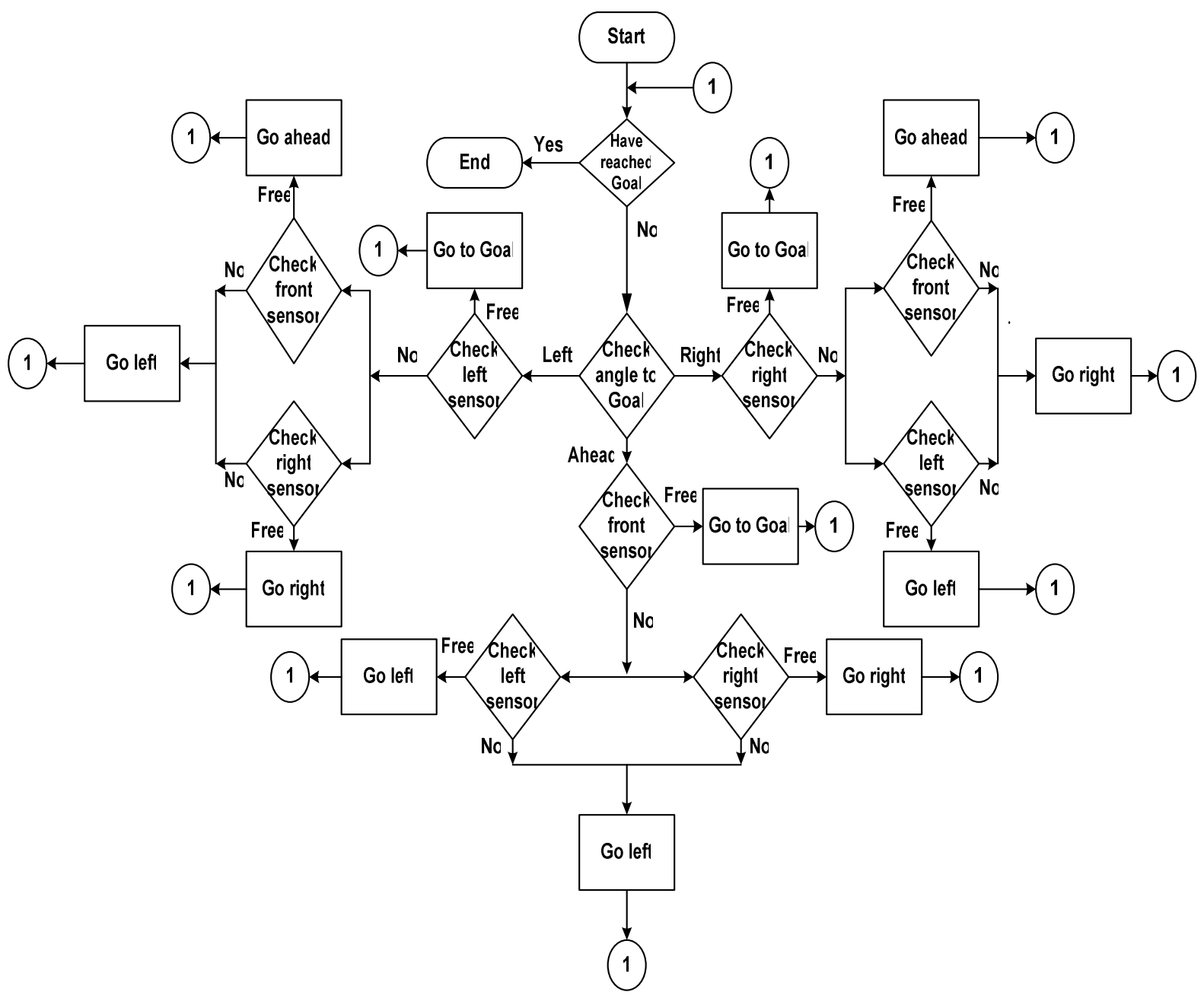

Fig. 14. Flow chart of the proposed planner. 


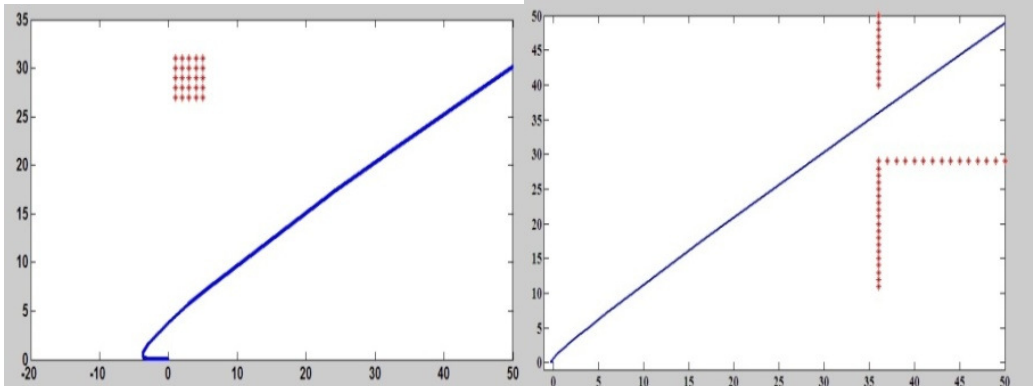

Initial angle $\square=$ zero Goal $x=50, y=30$
Initial angle $\square=$ zero

Goal $x=50, y=50$

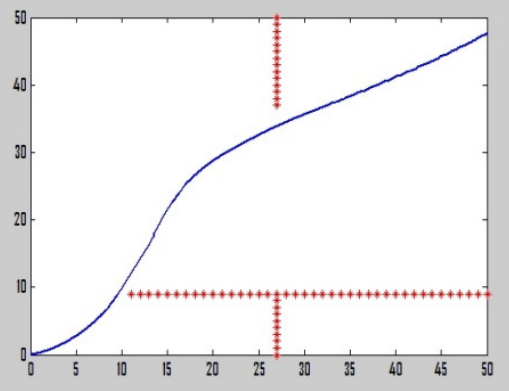

Initial angle $\square=$ zero

Goal $x=50, y=50$

Fig. 15.The simulation results for different environments with various obstacle distributions 